\title{
A Study of Taiwanese Design Student's Preference and Imagery for Textures of "Tujia brocade"
}

\author{
Wei Chow \\ Department of Industrial Design, National Cheng Kung \\ University, \\ Taiwan, ROC \\ E-mail:773469019@qq.com
}

\author{
Meng-Dar Shieh \\ Department of Industrial Design, National Cheng Kung \\ University \\ Taiwan, ROC
}

\begin{abstract}
Tujia minority is located at the border of four provinces including Hunan, Hubei, Sichuan, and Guizhou. With long-term historical and cultural influences, Tujia minority eventually formed its own unique style of folk craftworks, among which the "Tujia brocade" is one of the most representative art form. The research target in this study is the "Tujia brocade" textures within which six patterns are selected for investigation. The purpose is to investigate Taiwanese design students' preference and imagery for the "Tujia brocade" textures. This study was conducted with several approaches including documentary analyses, professional interviewing, and questionnaire surveys. Opinions available in references and discussions by professionals and scholars were collected for analysis and conclusion. Eleven samples for the questionnaire survey were selected by simple random sampling. The semanticdifference approach was used to design the questionnaire of imagery and the degree of preference. Some Taiwanese design students were selected as the questionnaire survey target and the effective sample size is 52 . The research results were arranged by using the descriptive statistics. It was found from the research results that, there are more apparent differences in the subjects' imagery perception from the "geometrical" and "regular" aspects. They incline to consist with each other from the "decorative" and "ethical" aspects. For the degree of preference, the subjects prefer the "Tujia brocade" textures which are more of the "animal-themed type", "plant-themed type", and "customand tradition-themed type".
\end{abstract}

Keywords-Taiwanese design student;Tujia brocade;preference and imagery

\section{INTRODUCTION}

Tujia minority is located at the border of four provinces including Hunan, Hubei, Sichuan, and Guizhou. With longterm historical and cultural influences, Tujia minority eventually formed its own unique style of folk craftworks, among which the "Tujia brocade" is one of the most representative art form. The research target in this study is the "Tujia brocade" textures within which six patterns are selected for investigation. The purpose is to investigate Taiwanese design students' preference and imagery for the "Tujia brocade" textures.

It is one of the four famous brocades in China. It is called
"Xilankapu" in Tujia ethnic language, "Tujia brocade" has a long history dating back more than 4000 years. It is a local people's folk craft from generation to generation. It reflects the local people's diligence and wisdom and it comes from their vivid lifestyle and gorgeous culture. Tujia brocade was included in the National minority non-material cultural heritage protection list in November 2005 in order to pass on and carry forward their excellent traditional culture. However, with the advances of economic and technology, these folk cultures has taken a huge impact and it has been faced with the risk of being ignored. Exploring the innovational direction on the basis of traditional craft techniques is inevitable. This study serves as a good reference for designers and cultural art creators during the design process and also provides a direction for the promotion and commercialization of the "Tujia brocade" in the Taiwan area.

\section{REFERENCE ANALYSIS}

\section{A. Plant-themed type}

In the process of getting along with the nature, Tujia people have tried to pick up a number of plant modeling and to apply to the creation of "Tujia brocade" patterns. Moreover, they are not only praising wintersweet, orchid, bamboo and chrysanthemum which are known as four gentlemen in Chinese classic literature, but also such as sunflower, aniseed, rose, lotus, chive, etc. It is fully embodies Tujia national characters like the enthusiasm for life and freedom. "Yan Qiang flower" is a pattern origining from stone walls in the period of their ancestors (see Fig.1). It is said that these stone walls looked like flowers opening in the gaps of rocks. Local individuals considered "Yan Qiang flower" to be a symbol of the tough character of Tujia people. In addition, the praise of wintersweet is quite universal since it represents elegance and nobleness. Tujia Weavers replaced wintersweet by rhombuses and squares since these two shapes coincide with the spiritual quality of wintersweet that is tenacious and courageous. "Da Lang Ku Mei" is a representative red- dominated pattern among them and its main body is nine wintersweet flowers arranged neatly with auspicious patterns around the principal part (see Fig.2). 


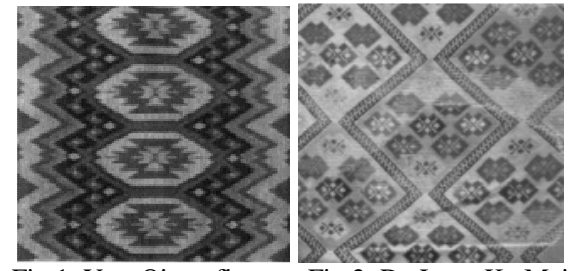

Fig.1. Yan Qiang flower Fig.2. Da Lang Ku Mei

\section{B. Animal-themed type}

Living in the mountains for a long time makes Tujia people observe animals rather carefully, thus "Tujia brocade" consists of a variety of animal-themed patterns, not only uses romantic expression to highlight animals' certain characteristics, but uses the two-dimensional expressive method to make the pattern more vivid and artistic. It is not uncommon such as "Ma Bi flower" (see Fig.3), "Monkey flower", "Tiger Footprints flower", "Die chi flower", "Yang Que flower" (see Fig.4), and other patterns.
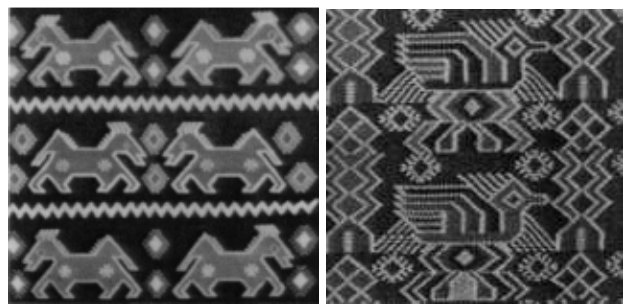

Fig.3. Ma Bi flower Fig.4. Yang Que flower

\section{Appliance-themed type}

Tujia people always so self-sufficient that they are deeply attached to chairs, tables, working tools which are frequentlyused in daily life. They applied these shapes of tools to tapestry design for showing their love for life. More representative of them are "Desk flower" (see Fig.5), "Chair flower", "Babajia flower" and so forth.

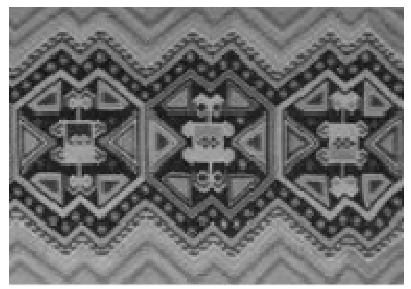

Fig.5. Desk flower

\section{Custom- and tration-themed type}

"Tujia brocade" patterns have quite abundant patterns reflecting folk customs, for instance "Ying Qin Tu" (see Fig. 6) and "Lao Shu Jia Nv" (see Fig.7). These patterns embody their enthusiasm and pride for their ethnic culture. As shown in the right drawing, it is a scene of wedding but it's for mice.
Weavers let the scene so lively and cheerful that it becomes more fascinating.
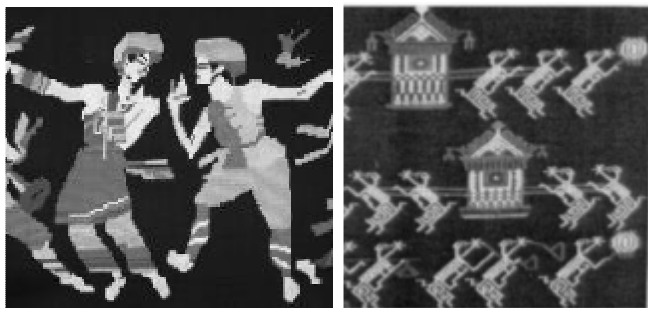

Fig.6. Ying Qin Tu Fig.7. Lao Shu Jia Nv

\section{E. Literature- and geometry- themed type}

In "Tujia brocade" patterns, "literature- and geometrythemed" patterns including typical "Single Eight Hooks flower ", "Double Eight Hooks flower", "Twenty-four Hooks flower" (see Fig.8), "Fourty-eight Hooks flower" and so forth which were created through local people's closely observation and deep understanding for them. Also, it shows local residents' strong and firm national spirits. "Single Eight Hooks flower" has strong expression and sense of balance. Common auspicious patterns like "Wan Zi Liu Shui flower" (see Fig.9), "Wang Zi flower", "Shou Zi flower","Yi Pin Dang Chao" and so on.

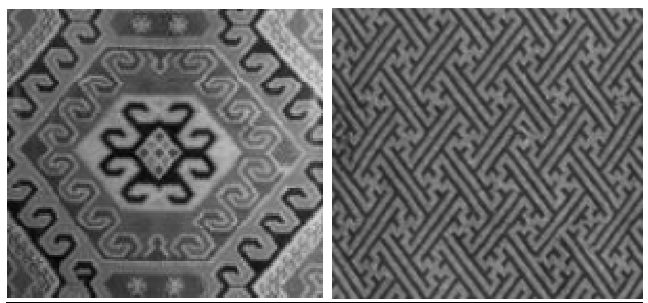

Fig.8. Twenty-four Hooks flower Fig.9. Wan Zi Liu Shui flower

\section{F. Modern-themed type}

Certain patterns of "Tujia Brocade" might be impacted by some cross-stitch decoration of the Qing Dynasty, like "Shuang Feng Chao Yang", "Lu Si Cai Lian" (see Fig.10). The right picture above is "Long Feng Wen" (see Fig.11) combining dragon's head, turtle's body and phoenix tail together to compose a sophisticated picture which is full of romantic colors and imagination.

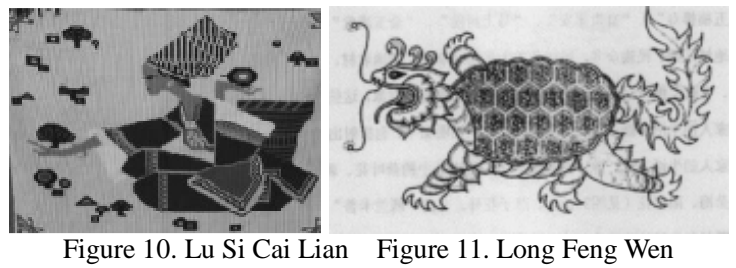

\section{METHODOLOGY}

This study was conducted with several approaches including documentary analyses, professional interviewing, 
and questionnaire surveys.

Firstly, 100 adjective vocabularies was collected, analyzed and summarized by condulting literature, relevant books, periodicals and experts' perspectives. Secondly, 40 vocabularies (see Table 1) were chosen from 100 adjective vocabularies to be the most appropriate words for organizing questionnaires .Then these 40 words were divided into 10

TABLE 1. 40 ADJECTIVE VOCABULARIES

\section{0 adjective vocabularies}

\begin{tabular}{|l|l|l|l|l|}
\hline geometry & continuous & constructive & homely & grunge \\
\hline historical & ancient & aesthetic & graceful & charming \\
\hline experienced & conventional & decorative & technic & skill \\
\hline religious & spiritual & mysterious & magical & national \\
\hline geographical & unique & totemic & marked & symbolic \\
\hline living & daily & harmony & regular & repeated \\
\hline lively & abundant & richly & cultural & emotional \\
\hline cultural & imaginative & narrative & allegoric & metaphorical \\
\hline
\end{tabular}

groups by inviting relevant researchers to conduct expert questionnaires. And then, experts were invited to extract a word from every group through the second expert questionnaire. In addition, 11 representative pictures were selected from related books and literatures. Next, the semantic- difference approach was used to design the following questionnaires of imagery and the degree of preference. Finally, 52 Taiwanese design students were selected as participants in this study.

Questionnaires took 11 samples and 10 adjective vocabularies as measure standards and contained 5 rating scales which were strongly disagree, disagree, in general, agree and totally agree. Participants were required to choose the most suitable option among them. These 5 options would be converted to 1 point, 2 points, 3 points, 4 points and 5 points.

Define abbreviations and acronyms the first time they are used in the text, even after they have been defined in the abstract. Abbreviations such as IEEE, SI, MKS, CGS, sc, dc, and rms do not have to be defined. Do not use abbreviations in the title or heads unless they are unavoidable.

\section{RESULTS AND DISCUSSIONS}

The chart (see Fig.12) is about "modern-themed" patterns and the sides of "cultural", "ethical" and "totemic" are more projecting.

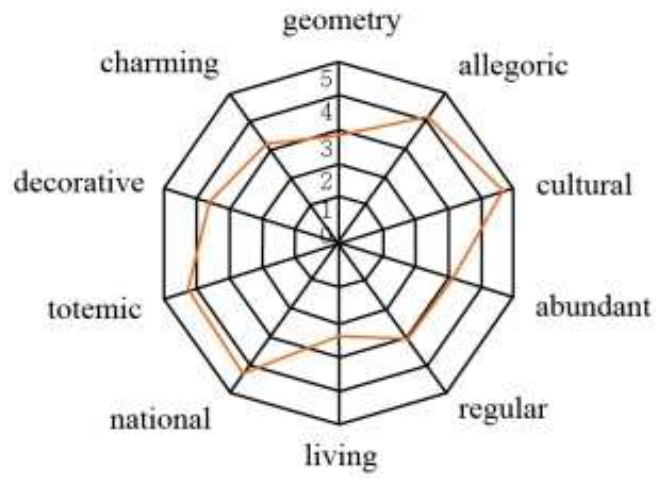

Fig. 12. A "modern-themed" pattern's imagery radar map
The picture (see Fig.13) regarding to "plant-themed" patterns are noticeable in the "geometrical", "totemic" and "regular" sides.

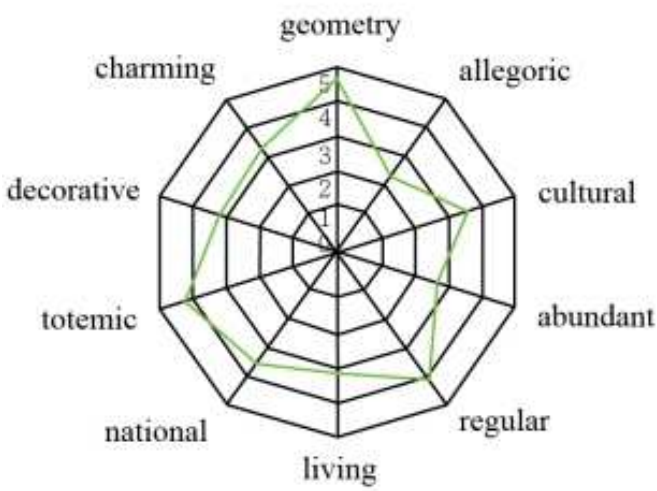

Fig. 13. A "plant-themed" pattern's imagery radar map

The chart relating to "literature- and geometry-themed" patterns is prominent in the fields of "geometrical" and "regular" (see Fig.14).

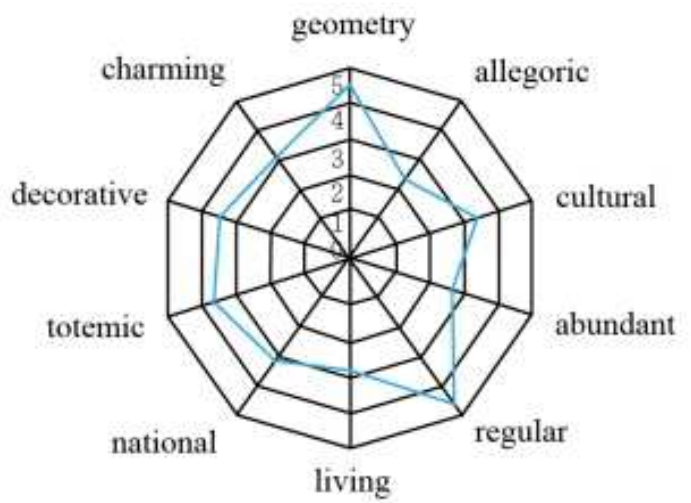

Fig. 14. A "literature- and geometry-themed" pattern's imagery radar map

The chart (see Fig.15) is regarding to "custom- and tradition-themed" patterns and according to the figure, the sides of "cultural", "ethical" and "meaningful" are more prominent.

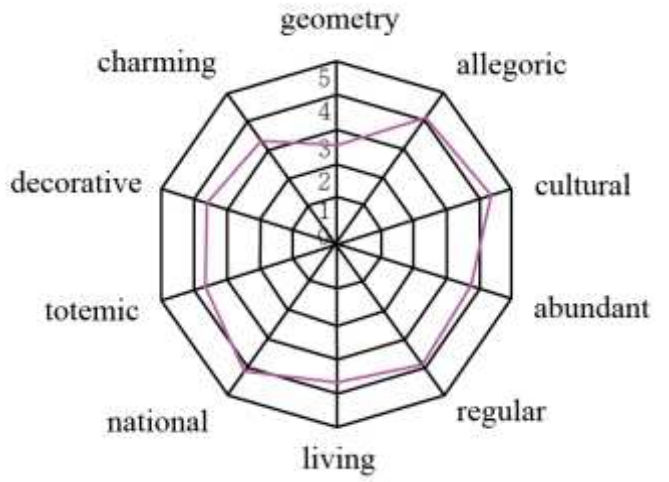

Fig. 15. A "custom- and tradition-themed" pattern's imagery radar map 
The figure (see Fig.16) is an "animal-themed" pattern's analysis map. The aspects of "regular", "geometrical", "totemic" are more prominent.

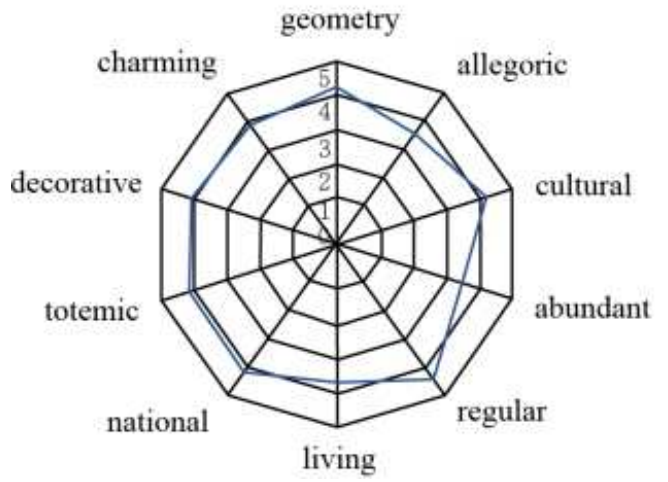

Fig. 16. An "animal- and tradition-themed" pattern's imagery radar map

The figure (see Fig.17) is an "animal-themed" pattern's analysis chart. According to the figure, the "regular", "geometrical", "decorative" aspects are more prominent. geometry

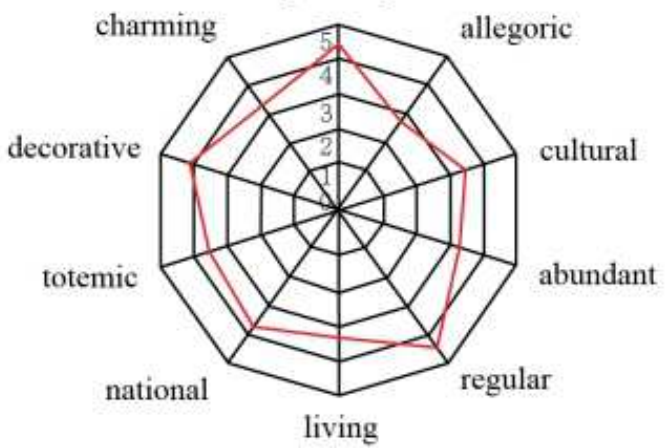

Fig.17. An “animal- and tradition-themed" pattern’s imagery radar map

This map (see Fig.18) is relating to "custom- and tradition-themed" patterns. From the above graph, these type of texture is noticeable in the aspects of "cultural", "ethical" and 'meaningful'.

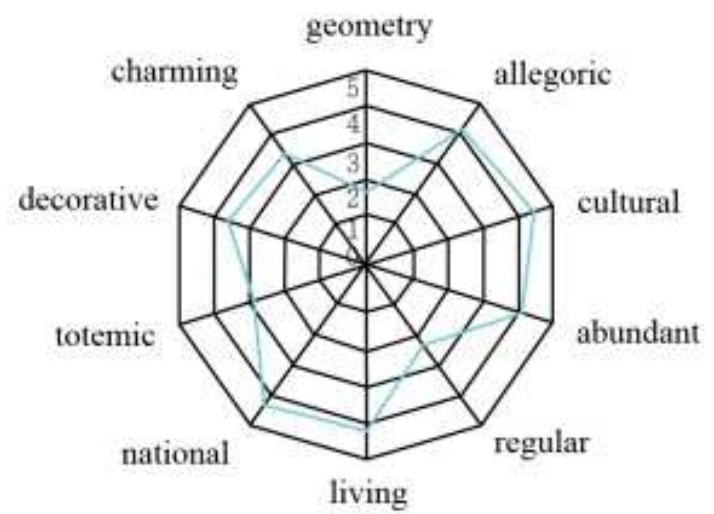

Fig.18. A “custom- and tradition-themed" pattern's imagery radar map

Fig.19 concerning "appliance-themed "patterns which is projecting in "geometrical" and "regular" aspects.

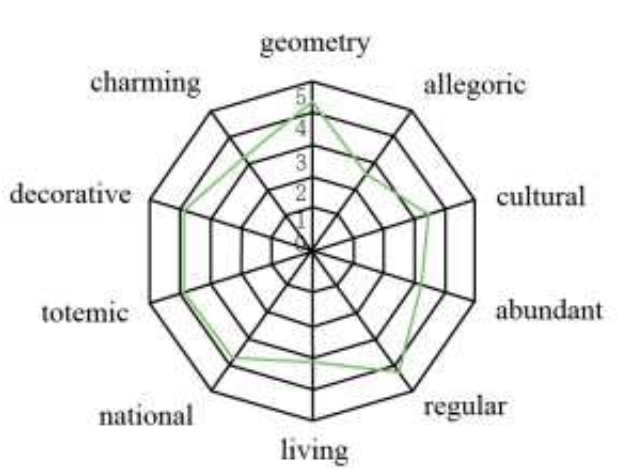

Fig.19. An "appliance-themed" pattern's imagery radar map

The figure (see Fig.20) is an "animal-themed" pattern's analysis graph. According to this picture, the aspect of "regular" is more prominent.

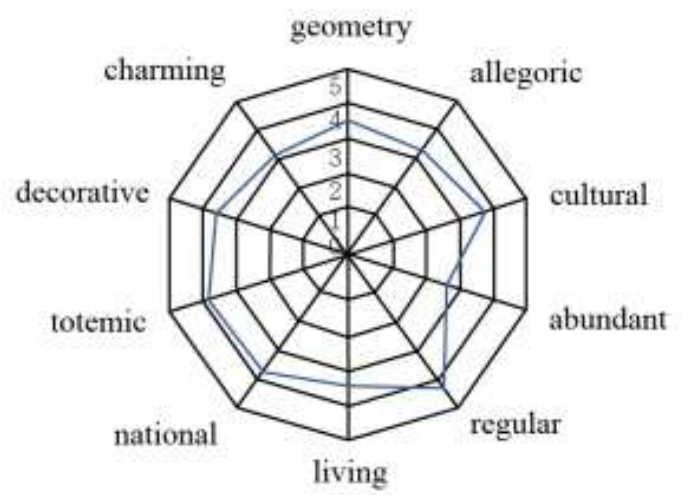

Fig.20. An “animal-themed” pattern's imagery radar map

The figure (see Fig.21) is a "modern-themed" texture's analysis graph. As shown above, it is outstanding in the aspects of "cultural", "ethical" and "decorative".

geometry

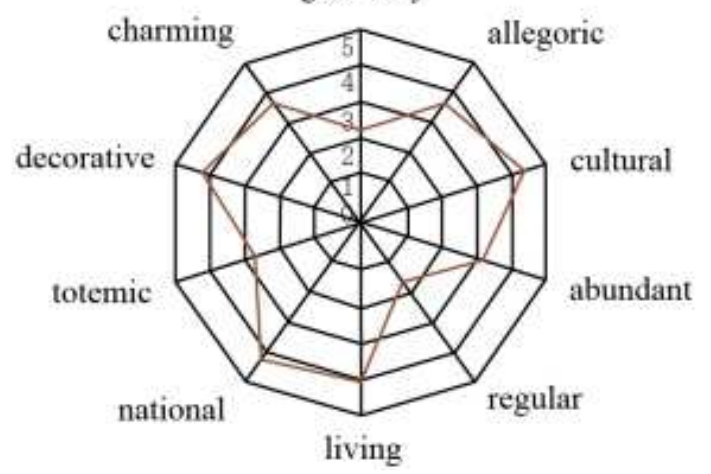

Fig.21. A “modern-themed" pattern’s imagery radar map

The figure (see Fig.22) is a "modern-themed" texture's analysis graph. As shown above, these type of texture is exceptional in "geometrical", "regular" and "totemic" aspects. 


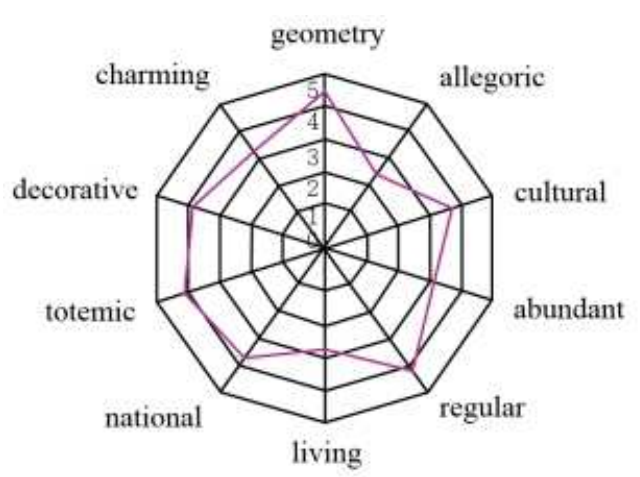

Fig. 22. A “modern-themed" pattern's imagery radar map

As Table 2 shown, this is a bar graph showing the degree of preference of some Taiwan design students about "Tujia brocade" patterns. Bar 5, bar 6 and bar 7 is higher in this picture and these bars respectively correspond to "Animalthemed", "Plant-themed" and "Custom- and tradition-themed" patterns. According to this, the subjects may prefer "animalthemed type", "plant- themed type", and "custom- and tradition-themed type" patterns among "Tujia brocade" patterns.

\section{TABLE 2. THE DEGREE OF PREFERENCE OF SOME TAIWAN DESIGN STUDENTS CONCERNING "TUJIA BROCADE" PATTERNS}

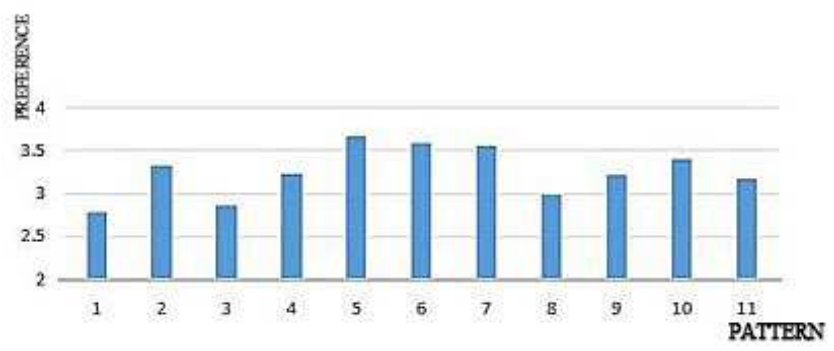

\section{CONCLUSION AND SUGGESTION}

The results of the study found that the image of the subjects in the "geometric" and "regular" aspects will produce more outstanding, and in the "decorative" and "national" aspects are more consistent. According to the degree of preference of students from department of design in Taiwan, It can be presumed that the subjects prefer to "Animal-themed type pattern", "Plant-themed type pattern" and "Custom- and tradition-themed type pattern".

This study serves as a good reference for designers and cultural art creators during the design process and also provides a direction for the promotion and commercialization of the "Tujia brocade" in the Taiwan area.

\section{Acknowledgment}

First of all, I would like to express my sincere heartfelt thanks to Professor Shieh and Professor Lin for them invaluable advice and constant encouragement. Then I would also like to express my gratitude to seniors and friends because they provide me with great convenience.

\section{References}

[1] Aileen, M.A., \& Maribeth, G.B. (2016). Managing Embroidery Business in Heritage Town of Taal, Batangas, Philippines. Asia Pacific Journal of Academic Research in Business Administration, Vol. 2 No. 1, April 2016.

[2] Hee, Y.K., \& Ji, S. H. (2016). Communication of Tactile Experiences in Handcraft. Online Published. August 31,2016.

[3] Katherine, B.U. (2016). Evolving Patterns: Conflicting Perceptions of Cultural reservation and the State of Batik's Cultural Inheritance Among Women Artisans in Guizhou, China. Lawrence University Honors Projects. Paper 97.

[4] Ran, H.F. (2008). The Craft Nature and Cultural Ecology of Tujia Embroidery. The Research Center for South Minorities.

[5] Yang, Y. (2016). Making up book section title Assessment of Cultural Tourism Experience in Selected Attraction in Nanyang, Henan Province in China. Journal of Tourism \& Hospitality Volume 5, Issue 2, 1000205. 